\title{
Conductance of quantum impurity models from quantum Monte Carlo
}

\author{
Dong E. Liu, Shailesh Chandrasekharan, and Harold U. Baranger \\ Department of Physics, Duke University, P.O. Box 90305, Durham, North Carolina 27708-0305, USA
}

(Received 30 July 2010; published 28 October 2010)

\begin{abstract}
The conductance of two Anderson impurity models, one with twofold and another with fourfold degeneracy, representing two types of quantum dots, is calculated using a world-line quantum Monte Carlo (QMC) method. Extrapolation of the imaginary time QMC data to zero frequency yields the linear conductance, which is then compared to numerical renormalization-group results in order to assess its accuracy. We find that the method gives excellent results at low temperature $\left(T \leqq T_{\mathrm{K}}\right)$ throughout the mixed-valence and Kondo regimes but it is unreliable for higher temperature.
\end{abstract}

DOI: 10.1103/PhysRevB.82.165447

PACS number(s): 72.10.Fk, 02.70.Ss, 73.21.La, 73.23.-b

Quantum dots provide a highly controlled and tunable way to study a range of quantum many-body physics: various quantum impurity models and their associated Kondo effects, ${ }^{1-6}$ tunneling with dissipation, ${ }^{7}$ and Luttinger liquid effects, ${ }^{8,9}$ to name a few. The crucial experimental observable in these situations is the conductance; thus, calculating the conductance is a key task for both analytic and numerical approaches. Numerical methods have indeed been developed, ${ }^{10-13}$ with remarkable agreement for small systems between theory and experiment. ${ }^{14}$ But these methods scale poorly for the larger, more complex multidot systems ${ }^{15,16}$ that are currently of great interest. Here we implement and test a way to calculate the conductance from a path-integral quantum Monte Carlo (QMC) calculation. While it yields less information than numerical renormalization group (NRG) in simple systems (e.g., a single quantum dot), the method should scale readily to more complicated systems. Results for two Anderson-type impurity models show that the method works very well at low temperature.

For calculations of the conductance in simple quantum dot systems, the most accurate results are obtained using the NRG method. ${ }^{10-13}$ NRG becomes slow and even impractical, however, if there are many leads, a many-fold degeneracy, or more than a few interacting sites. In such situations, the world-line QMC method could be a valuable alternative since it scales nicely as the problem size increases. However, QMC is formulated in imaginary time rather than real time: to extract dynamic properties one must transform from imaginary back to real time. The statistical error in the QMC data makes this an ill-posed problem, for which various extrapolation and continuation methods have been developed. ${ }^{17}$ To obtain the conductance of interest here, we extrapolate to zero frequency the appropriate correlation function evaluated using QMC at the imaginary Matsubara frequencies. ${ }^{18-22}$ This has been used, for instance, to study a one-dimensional (1D) Hubbard chain coupled to noninteracting leads in the absence of the Kondo effect. ${ }^{22}$

The aim of this paper is to test the validity of the extrapolation method for Anderson impurity models in both the mixed-valence and Kondo regimes. We study the linear conductance using QMC in two models: a single impurity Anderson model with either twofold or fourfold degeneracy. The standard twofold degenerate model is a simplified representation of a single GaAs quantum dot connected to leads. ${ }^{1}$ The fourfold degenerate model represents a quantum dot in a carbon nanotube in which there is an additional orbital degeneracy from the helicity of the states. ${ }^{1-4}$ This orbital degeneracy is present in both the discrete states in the dot and the extended states in the carbon nanotube leads.

Consider a model, then, in which a single level with Coulomb repulsion $U$ represents the quantum dot (which we also refer to as the impurity site) and is coupled to two noninteracting bands, left $(L)$ and right $(R)$. The degeneracy of both the discrete level and the free electrons is $M$; we will consider the two cases $M=2$ (standard single-level Anderson model) and $M=4$ (both spin and orbital degeneracy). The Hamiltonian is

$$
\begin{aligned}
H= & \sum_{k, i=\{L, R\}} \sum_{\sigma=1}^{M} \epsilon_{k} c_{k i \sigma}^{\dagger} c_{k i \sigma}+\frac{U}{2}\left(\hat{N}-N_{g}\right)^{2} \\
& +\sum_{k, i=\{L, R\}} \sum_{\sigma=1}^{M} V_{\sigma}\left(c_{k i \sigma}^{\dagger} d_{\sigma}+\text { H.c. }\right),
\end{aligned}
$$

where the electron number operator for the impurity site is $\hat{N}=\sum_{\sigma=1}^{M} d_{\sigma}^{\dagger} d_{\sigma}$. The energy in the bands is such that $-D \leq \epsilon_{k}$ $\leq D$ where $D$ is the half bandwidth, and we assume a flat density of states, $\rho=1 / 2 D$. The hybridization of the impurity to each lead is given by $V_{\sigma}$ which yields a level width $\Gamma_{\sigma}=\Gamma_{L, \sigma}+\Gamma_{R, \sigma}$ with $\Gamma_{L, \sigma}=\Gamma_{R, \sigma}=\pi V_{\sigma}^{2} \rho$. In terms of the gate voltage $N_{g}$ (i.e., the equilibrium occupancy of the dot), the energy level of the dot is explicitly given by $\epsilon_{d}$ $=U\left(1-2 N_{g}\right) / 2$. Finally, in the absence of any orbital degeneracy, the degeneracy of the $d$ level is simply given by spin, $\sigma=\uparrow$ or $\downarrow$.

Method. A new basis for the two noninteracting bands can be independently constructed by starting from the localized impurity state. In this way the model is mapped to a 1D infinite tight-binding chain, ${ }^{10}$ as shown in Fig. 1. We use a large chain $\left(\sim 10^{6}\right.$ sites $)$ in order that its finite size is irrelevant for the physics of interest. Then, in order to make the computation time manageable, logarithmic blocking of the

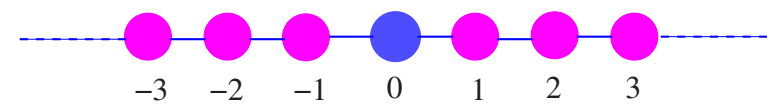

FIG. 1. (Color online) The 1D infinite tight-binding chain, where the zeroth site is the impurity site (quantum dot). 
energy levels is used to reduce the number of effective sites (in other words, we map the problem to a "Wilson chain"10). In this work, the logarithmic blocking factor is $\Lambda=2.5$ (the number of effective sites is $\sim 61)$. We use a form of blocking $^{23}$ which avoids $\Lambda$-dependent corrections ${ }^{24}$ to the low-energy scales [i.e., $\left.T_{\mathrm{K}}(\Lambda)\right]$. We solve the resulting problem using the world-line quantum Monte Carlo method with a directed-loop cluster algorithm. ${ }^{25,26}$ The Trotter number $N$ is chosen such that $\varepsilon=\beta / N \simeq 0.1 / D$.

To find the conductance, we proceed following the method of Syljuåsen in Ref. 22 which is itself closely related to several other approaches. ${ }^{18-21}$ The conductance at the (imaginary) Matsubara frequencies, $g\left(i \omega_{n}\right)$ with $\omega_{n}=2 \pi n T$, is related in linear response to the current-current correlation function in the usual way. For a one-dimensional system with open boundary conditions, current continuity can be used $^{19,20,22}$ to express $g\left(i \omega_{n}\right)$ in terms of charge correlations (polarizability),

$$
g\left(i \omega_{n}\right)=\frac{\omega_{n}}{\hbar} \int_{0}^{\beta} d \tau \cos \left(\omega_{n} \tau\right)\left\langle P_{x}(\tau) P_{y}(0)\right\rangle,
$$

where $P_{y}$ is the sum of the electron charge-density operators to the right of $y, P_{y} \equiv \sum_{y^{\prime} \geq y} \hat{n}_{y^{\prime}}$. Thus the time derivative of $\left\langle P_{y}\right\rangle$ is the current through the bond between sites $y-1$ and $y$. We calculate $g\left(i \omega_{n}\right)$ for $n>0$ from the world-line QMC data in this way. Not all combinations of $x$ and $y$ can be used in Eq. (2) because the system is not a physical chain but only effectively mapped to a chain. Notice that the current through the four bonds closest to the impurity site (labeled 0 ) corresponds to the physical current. Therefore, $x$ and $y$ must be chosen from among $\{-1,0,1,2\}$. In addition, left-right symmetry reduces the number of independent combinations. In our calculation, we choose three cases for $x$ and $y$ : $(0,1)$, $(0,0)$, and $(-1,0)$.

The linear conductance $G$ is obtained by extrapolating $g\left(i \omega_{n}\right)$ to zero frequency, $G=\lim _{\omega_{n} \rightarrow 0} g\left(i \omega_{n}\right)$. We carry out this extrapolation as follows. First, we try to fit the data at the four or five lowest Matsubara frequencies $\left[g\left(i \omega_{n}\right)\right.$ for $n$ $=1, \ldots, 4$ or $1, \ldots, 5]$ to a linear or quadratic polynomial. If this method yields a good fit, we simply extrapolate the data by using the polynomial. If neither polynomial fit is good, the data at the first 14 lowest Matsubara frequencies are fit by using a series of rational polynomial functions of different degree $[p / q]$ (e.g., $p$ for the numerator, $q$ for the denominator, $p=1$ for a constant, $p=2$ for linear function, etc.) as described in Ref. 22. We use all $p$ and $q$ such that $5 \leq p+q$ $\leq 10$ and $p, q \geq 2$ but exclude cases in which spurious poles appears. The final extrapolated value is the average of the results for these different forms, and the error bar at zero frequency is the maximum spread, which is larger than the error bar of any single $[p / q]$ extrapolation. To justify this method, we check that three conditions are met. (i) The data for all the combinations of $x$ and $y$ must extrapolate to nearly the same value (the current through different bonds at nonzero frequency can be different but current continuity requires that at zero frequency the current through all bonds be the same). (ii) The data should fit well to most of the functional forms of degree $[p / q]$ (we cannot exclude too many

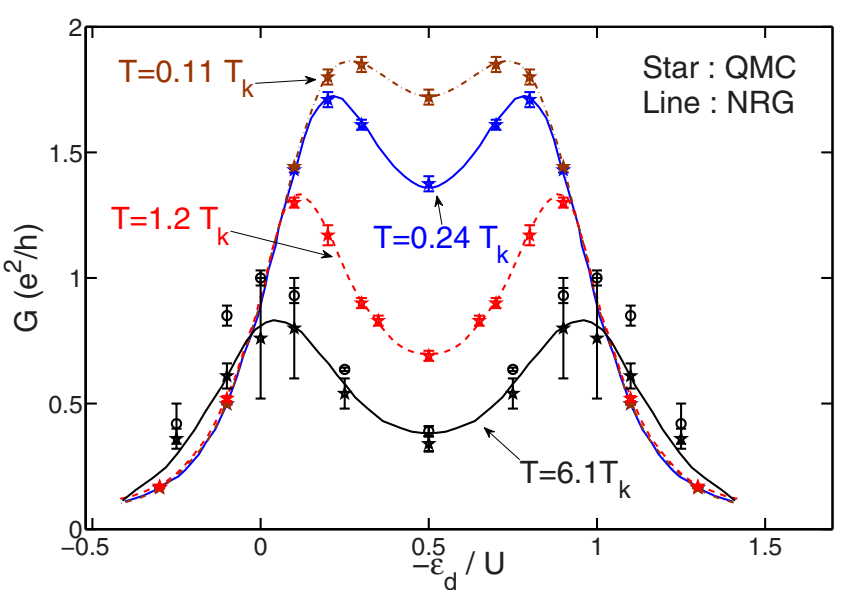

FIG. 2. (Color online) Conductance through a single-level Anderson model without orbital degeneracy as a function of gate voltage: QMC result (symbols) compared to NRG calculation (Ref. 13) (lines). Data for four temperatures are shown: $T \simeq 0.11 T_{\mathrm{K}}$ (brown, dot-dashed, $\beta=98.3$ ), $T \simeq 0.24 T_{\mathrm{K}}$ (blue, upper solid, $\beta$ =43.7), $T \simeq 1.2 T_{\mathrm{K}}($ red, dashed, $\beta=8.6)$, and $T \simeq 6.1 T_{\mathrm{K}}$ (black, lower solid, $\beta=1.7)$. For $T \simeq 6.1 T_{\mathrm{K}}$, the black stars are for $(x, y)$ $=(0,1)$ while the black circles are for $(0,0) . T_{\mathrm{K}}$ denotes the Kondo temperature found by NRG (Ref. 13) at the particle-hole symmetric point $\left(-\epsilon_{d} / U=0.5\right)$. Note the high accuracy of the QMC result as long as $T \lesssim T_{\mathrm{K}}$.

cases). (iii) Finally, the conductance should have a small error bar (a large error bar shows that the extrapolation is model dependent)

Conductance without orbital degeneracy. We first consider the standard single-level Anderson model, $M=2$ in Eq. (1). We compare the conductance obtained by our QMC calculation to that from the NRG calculation of Ref. 13 [see their Fig. 2(a)]. The parameters are $D=100, \Gamma=1.0$, and $U$ $=3 \pi$. The NRG value ${ }^{13}$ for the Kondo temperature at the particle-hole symmetry point $(-\epsilon / U=0.5)$, which we denote $T_{\mathrm{K}}$ throughout, is $T_{\mathrm{K}} \simeq 0.1$.

Figure 2 compares our calculation of the conductance as a function of gate voltage to the NRG results ${ }^{13}$ for several temperatures. The QMC results are in excellent agreement with the $N R G$ results for $T \leq T_{\mathrm{K}}$ for all values of the gate voltage - that is, in both the mixed-valance and Kondo regimes. For $T$ slightly larger than $T_{\mathrm{K}}$, agreement is good; in contrast, note that there is a substantial error in the extrapolated conductance value for larger $T$.

Some examples of the extrapolations used to obtain the conductance shown in Fig. 2 are given in Figs. 3-5, moving from lower to higher temperature. Figure 3 shows four examples of the conductance at imaginary frequency, $g\left(i \omega_{n}\right)$, for $T<T_{\mathrm{K}}$. Examples of a linear fit [panel (a)], a quadratic fit [panel (c)], and rational polynomial fits [panels (b) and (d)] are shown. In the mixed valance regime, $-\epsilon_{d} / U<0.1$ or $>0.9$, a linear or quadratic polynomial works well, and the three curves for different $(x, y)$ all extrapolate to nearly the same value, leading to a small error bar. In the Kondo regime, $0.1<-\epsilon_{d} / U<0.9$, the linear or quadratic polynomial does not fit well but the QMC data can be fit to a series of rational polynomials as discussed above. Almost all values of $[p / q]$ work well, and the three sets of $g\left(i \omega_{n}\right)$ for different 

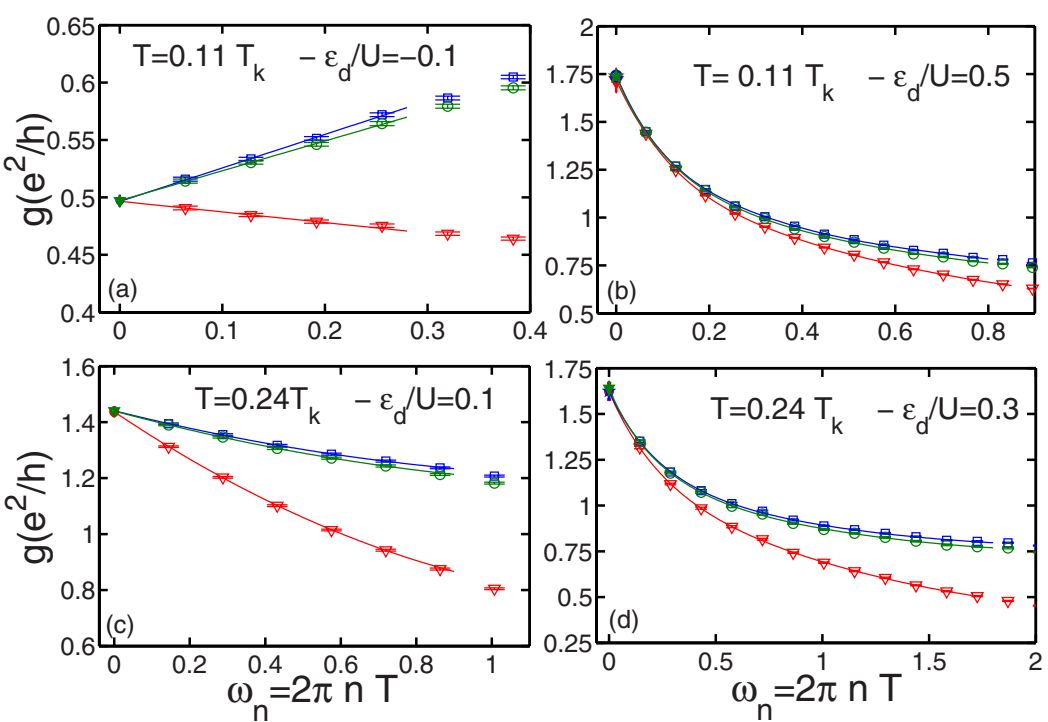

FIG. 3. (Color online) Conductance at Matsubara frequencies at low temperature (symbols) for the single-level Anderson model without orbital degeneracy and the corresponding fits used to extrapolate to zero frequency (lines). The values of $T$ and $-\epsilon / U$ are (a) $0.11 T_{\mathrm{K}},-0.1$; (b) $0.11 T_{\mathrm{K}}, 0.5$; (c) $0.24 T_{\mathrm{K}}, 0.1$; and (d) $0.24 T_{\mathrm{K}}, 0.3$. Points for three choices of $(x, y)$ are shown: $(0,1)$ red triangles, $(0,0)$ blue squares, and $(-1,0)$ green circles. A good quality extrapolation is obtained in all cases. $(x, y)$ extrapolate to nearly the same value, leading to a small error bar. In this temperature regime, then, the extrapolation is straight forward and the agreement with the NRG result is excellent.

For $T \sim T_{\mathrm{K}}$, two examples of the conductance function $g\left(i \omega_{n}\right)$ are shown in Fig. 4. For the mixed valance regime [Fig. 4(a)], a quadratic polynomial works well for $(x, y)$ $=(0,0)$ and $(-1,0)$, and rational polynomials are used for $(0,1)$. All three combinations extrapolate to nearly the same value, so the result is accurate. In the Kondo regime [panel (b), $\left.-\epsilon_{d} / U=0.5\right], g\left(i \omega_{n}\right)$ for $(0,1)$ can be fit with rational polynomials. However, for both other cases, $(x, y)=(0,0)$ and $(-1,0)$, there is a small wiggle near $\omega=2 \pi T_{\mathrm{K}}$ in the imaginary frequency conductance function $g\left(i \omega_{n}\right)$, showing that there is important structure below that frequency. Since there is only one data point below $\omega=2 \pi T_{\mathrm{K}}$, the extrapolation is unreliable. Thus, we do not use the data when structure appears at a frequency below which there are only a few
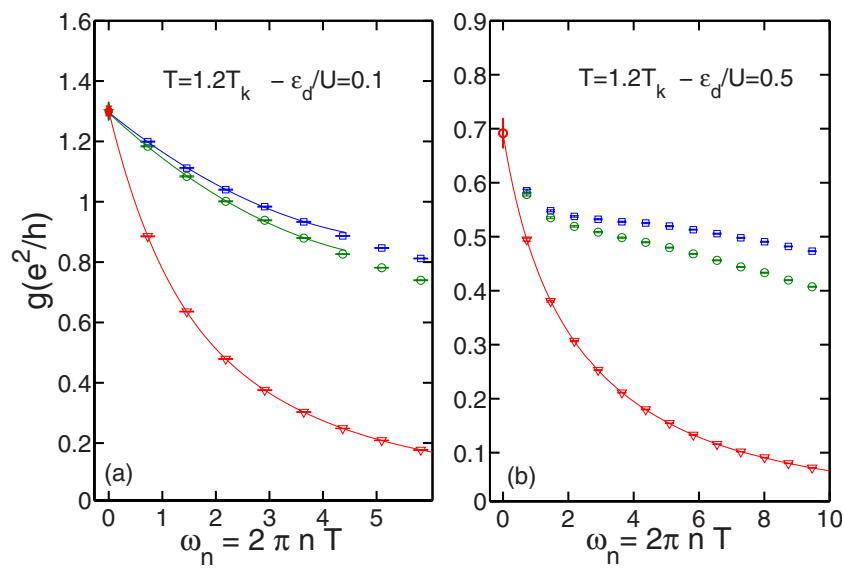

FIG. 4. (Color online) Conductance at Matsubara frequencies for $T \simeq 1.2 T_{\mathrm{K}}$ in the absence of orbital degeneracy (symbols) and the corresponding fits used to extrapolate to zero frequency (lines). The values of $-\epsilon / U$ are (a) 0.1 (mixed-valence) and (b) 0.5 (Kondo regime). Points for three choices of $(x, y)$ are shown: $(0,1)$ red triangles, $(0,0)$ blue squares, and $(-1,0)$ green circles. Extrapolation using $(x, y)=(0,1)$ is accurate. data points. The conductance in the Kondo regime for this temperature is based only on $(x, y)=(0,1)$; nonetheless, the agreement with the NRG result is good.

Finally, for $T>T_{\mathrm{K}}$ (Fig. 5), the functions $g\left(i \omega_{n}\right)$ for the three combinations of $(x, y)$ do not extrapolate to the same zero-frequency value. Notice also that the conductance obtained in the mixed-valence regime (the gate voltage at which the conductance peaks for this temperature) has a large error bar. For the cases $(x, y)=(0,0)$ and $(-1,0)$, the QMC data can be fit with a rational polynomial but the extrapolated result disagrees substantially with NRG. For the case $(0,1)$, the average value of $G$ from QMC roughly follows the NRG result (Fig. 2) but the large error bar in most cases indicates that the result has little meaning. Thus, the QMC extrapolation method is unreliable for $T$ substantially larger than $T_{\mathrm{K}}$.

Conductance with orbital degeneracy. We now turn to considering an Anderson model in which all the states, both
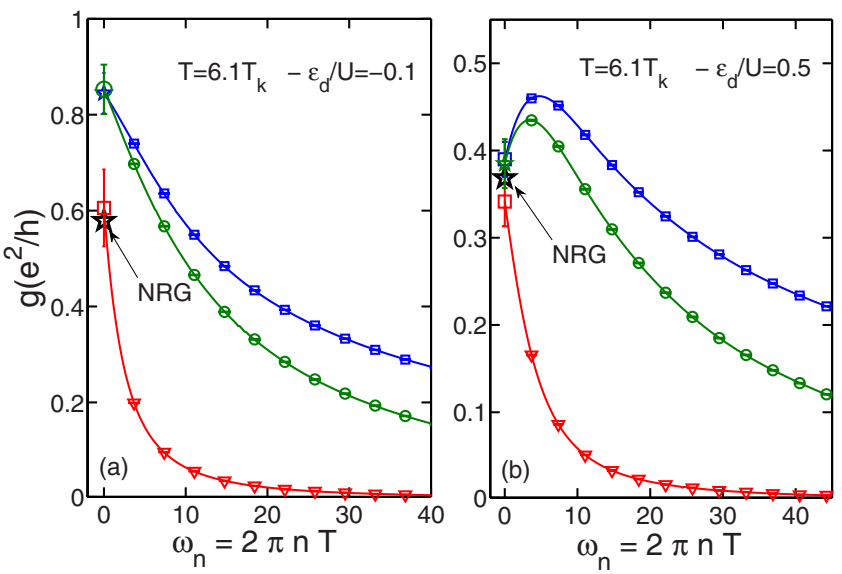

FIG. 5. (Color online) Conductance at Matsubara frequencies for high temperature, $T \simeq 6.1 T_{\mathrm{K}}$, in the absence of orbital degeneracy (symbols) and the corresponding fits used to extrapolate to zero frequency (lines). (a) $-\epsilon / U=-0.1$ and (b) $-\epsilon / U=0.5$. Points for three choices of $(x, y)$ are shown: $(0,1)$ red triangles, $(0,0)$ blue squares, and $(-1,0)$ green circles. The black stars are the NRG data. The accuracy of the extrapolation is poor in all of these cases. 


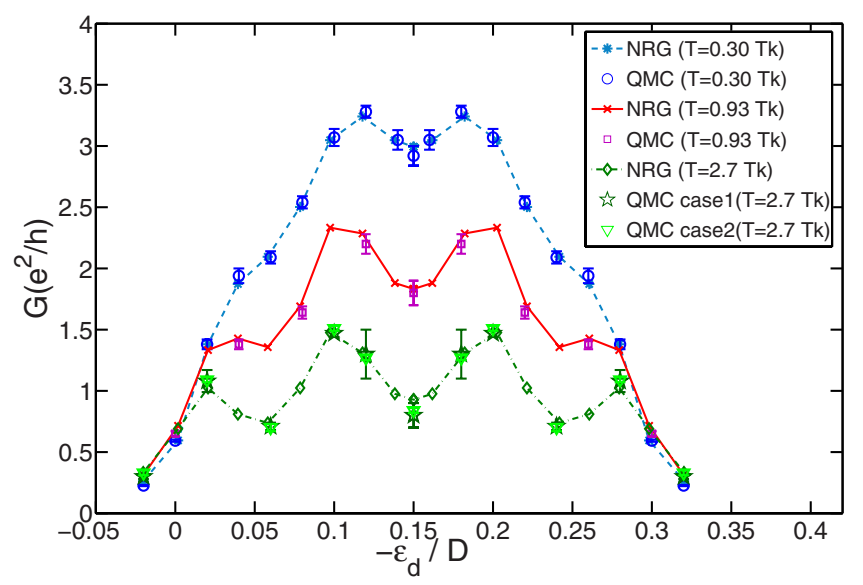

FIG. 6. (Color online) Conductance in fourfold degenerate model as a function of gate voltage: QMC results (symbols) compared with NRG calculations (Ref. 12) (lines with symbols). Results for three temperatures are shown: $T \simeq 0.30 T_{\mathrm{K}}$ (blue circles and solid line, $\beta=79.4$ ), $0.93 T_{\mathrm{K}}$ (red squares and dashed line, $\beta=25.6$ ), and $2.7 T_{\mathrm{K}}$ (green triangles or stars and dotted line, $\beta=8.77$ ). For the highest temperature $\left(T \simeq 2.7 T_{\mathrm{K}}\right)$, the QMC data labeled case 1 are based on $(x, y)=(0,0)$ and $(-1,0)$ while those for case 2 use $(0,1)$. $T_{\mathrm{K}}$ here denotes the Kondo temperature found by NRG (Ref. 12) at the particle-hole symmetric point. The good agreement of the QMC data with the NRG results illustrates the value of the QMC approach, though note the growing error bar when $T \gtrsim T_{\mathrm{K}}$.

those in the dot and in the leads, have an orbital degeneracy in addition to spin degeneracy: $M=4$ in Eq. (1). This situation arises, for instance, in carbon nanotube quantum dots connected to carbon nanotube leads..$^{2-4,27}$ To assess the quality of our QMC results, we compare with the NRG results of Ref. 12 (see their Fig. 16). The parameters we use are $D$ $=30, U=0.1 D=3, \Gamma_{1,2}=0.003 \pi D$, and $\Gamma_{3,4}=0.002 \pi D$. At the particle-hole symmetric point where the Kondo temperature is a minimum, the NRG estimation ${ }^{12}$ for $T_{\mathrm{K}}$ yields $T_{\mathrm{K}} \simeq 0.0014 D$.

Figure 6 compares our calculation of the conductance as a function of gate voltage to the NRG (Ref. 12) results. For $T \leq T_{\mathrm{K}}$, the QMC and NRG results are in very good agreement throughout both the mixed-valence and Kondo regimes. For $T>T_{\mathrm{K}}\left(T \simeq 2.7 T_{\mathrm{K}}\right)$, the $\mathrm{QMC}$ conductance roughly follows the NRG result but does not accurately agree with it. In addition, a large error bar is encountered at the highest temperature, showing that, as in the doubly degenerate case, the extrapolation is not reliable for these temperatures.

Four examples of the extrapolation from the imaginary frequency conductance function, $g\left(i \omega_{n}\right)$, are shown in Fig. 7. At low temperature, panel (a), the extrapolation is good and consistent for all three values of $(x, y)$ using the rational polynomial fit. Near the particle-hole symmetry point and for
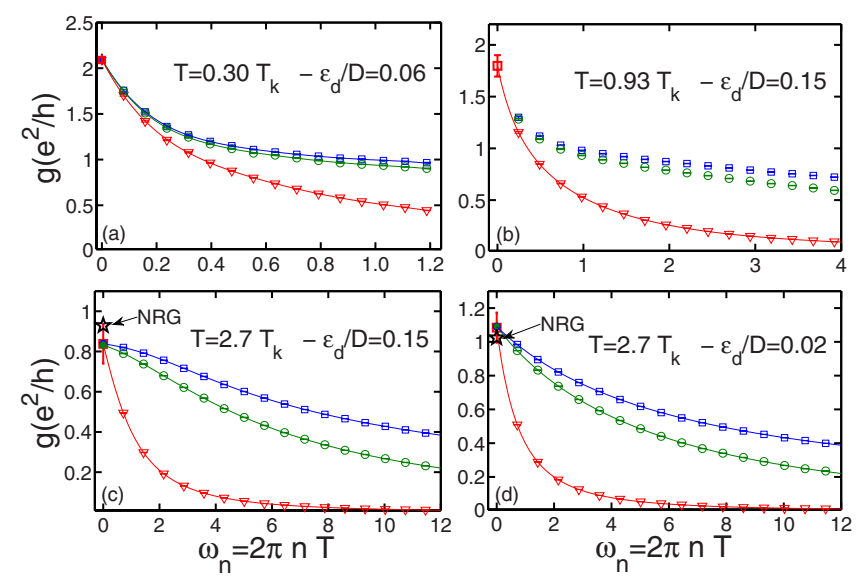

FIG. 7. (Color online) Imaginary frequency conductance function for single impurity Anderson model with orbital degeneracy $(M=4)$. The values of $T$ and $-\epsilon / U$ are (a) $0.30 T_{\mathrm{K}}, 0.06$, (b) $0.93 T_{\mathrm{K}}$, 0.15 (near particle-hole symmetry), (c) $2.7 T_{\mathrm{K}}, 0.15$, and (d) $2.7 T_{\mathrm{K}}$, 0.02 . Points for three choices of $(x, y)$ are shown: $(0,1)$ red triangles, $(0,0)$ blue squares, and $(-1,0)$ green circles. The black stars are the NRG data. The extrapolation is successful at low temperature but becomes increasingly problematic at higher temperature, $T>T_{\mathrm{K}}$.

$T \simeq T_{\mathrm{K}}$ [panel (b)], the case with $(x, y)=(0,1)$ fits nicely to a rational polynomial and the extrapolated value agrees with NRG. For the other two curves $[(x, y)=(0,0)$ and $(-1,0)]$, a small wiggle appears near $\omega \simeq 2 \pi T_{\mathrm{K}}$, as in the case without orbital degeneracy (Fig. 4), making extrapolation difficult. For larger $T$, panels (c) and (d), although the QMC data for two cases $[(x, y)=(0,0)$ and $(-1,0)]$ can be fit to rational polynomials and yield an estimated conductance with small error bar, the value does not agree accurately with the NRG result. The $(x, y)=(0,1)$ yields a large estimated error. Therefore, as we saw in the case without orbital degeneracy, when the temperature become large, the QMC method becomes inaccurate.

In summary, we developed and tested a method to obtain the linear conductance by extrapolating from QMC data. By studying two cases for which NRG results exist in the literature, ${ }^{12,13}$ we demonstrated the accuracy of the extrapolation technique as long as the temperature is not too high, $T \lesssim T_{\mathrm{K}}$ (where $T_{\mathrm{K}}$ denotes the Kondo temperature at the particle-hole symmetric point). We expect that this technique will be useful for finding the conductance of more complex quantum dot and/or impurity systems, such as three and four quantum dot structures. ${ }^{15,16}$

We thank P. Bokes and J. Shumway for helpful discussions. This work was supported in part by the U.S. NSF under Grant No. DMR-0506953. 
${ }^{1}$ M. Grobis, I. G. Rau, R. M. Potok, and D. Goldhaber-Gordon, in Handbook of Magnetism and Advanced Magnetic Materials, edited by H. Kronmüller and S. Parkin (Wiley, New York, 2007), Vol. 5.

${ }^{2}$ P. Jarillo-Herrero, J. Kong, H. S. van der Zant, C. Dekker, L. P. Kouwenhoven, and S. De Franceschi, Nature (London) 434, 484 (2005).

${ }^{3}$ A. Makarovski, A. Zhukov, J. Liu, and G. Finkelstein, Phys. Rev. B 75, 241407 (2007).

${ }^{4}$ A. Makarovski, J. Liu, and G. Finkelstein, Phys. Rev. Lett. 99, 066801 (2007).

${ }^{5}$ R. M. Potok, I. G. Rau, H. Shtrikman, Y. Oreg, and D. Goldhaber-Gordon, Nature (London) 446, 167 (2007).

${ }^{6}$ N. Roch, S. Florens, T. A. Costi, W. Wernsdorfer, and F. Balestro, Phys. Rev. Lett. 103, 197202 (2009).

${ }^{7}$ Y. Bomze, H. Mebrahtu, I. Borzenets, A. Makarovski, and G. Finkelstein, Phys. Rev. B 79, 241402 (2009).

${ }^{8}$ C. Livermore, C. H. Crouch, R. M. Westervelt, K. L. Campman, and A. C. Gossard, Science 274, 1332 (1996).

${ }^{9}$ D. Berman, N. B. Zhitenev, R. C. Ashoori, and M. Shayegan, Phys. Rev. Lett. 82, 161 (1999).

${ }^{10}$ K. G. Wilson, Rev. Mod. Phys. 47, 773 (1975).

${ }^{11}$ R. Bulla, T. A. Costi, and T. Pruschke, Rev. Mod. Phys. 80, 395 (2008).

${ }^{12}$ W. Izumida, O. Sakai, and Y. Shimizu, J. Phys. Soc. Jpn. 67, 2444 (1998)
${ }^{13}$ T. A. Costi, Phys. Rev. B 64, 241310 (2001).

${ }^{14}$ A. C. Seridonio, M. Yoshida, and L. N. Oliveira, EPL 86, 67006 (2009).

${ }^{15}$ K. Grove-Rasmussen, H. I. Jrgensen, T. Hayashi, P. E. Lindelof, and T. Fujisawa, Nano Lett. 8, 1055 (2008).

${ }^{16}$ L. Gaudreau, A. Kam, G. Granger, S. A. Studenikin, P. Zawadzki, and A. S. Sachrajda, Appl. Phys. Lett. 95, 193101 (2009).

${ }^{17}$ M. Jarrell and J. E. Gubernatis, Phys. Rep. 269, 133 (1996).

${ }^{18}$ O. Sakai, S. Suzuki, W. Izumida, and A. Oguri, J. Phys. Soc. Jpn. 68, 1640 (1999).

${ }^{19}$ K. Louis and C. Gros, Phys. Rev. B 68, 184424 (2003).

${ }^{20}$ P. Bokes and R. W. Godby, Phys. Rev. B 69, 245420 (2004).

${ }^{21}$ M. J. Verstraete, P. Bokes, and R. W. Godby, J. Chem. Phys. 130, 124715 (2009).

${ }^{22}$ O. F. Syljuåsen, Phys. Rev. Lett. 98, 166401 (2007).

${ }^{23}$ V. L. Campo and L. N. Oliveira, Phys. Rev. B 72, 104432 (2005).

${ }^{24}$ H. R. Krishna-murthy, J. W. Wilkins, and K. G. Wilson, Phys. Rev. B 21, 1003 (1980).

${ }^{25}$ O. F. Syljuåsen and A. W. Sandvik, Phys. Rev. E 66, 046701 (2002).

${ }^{26}$ J. Yoo, S. Chandrasekharan, R. K. Kaul, D. Ullmo, and H. U. Baranger, Phys. Rev. B 71, 201309(R) (2005).

${ }^{27}$ M.-S. Choi, R. López, and R. Aguado, Phys. Rev. Lett. 95, 067204 (2005). 